\title{
Set-valued differentials and a nonsmooth version of Chow's Theorem*
}

\author{
Franco Rampazzo \\ Dipartimento di Matematica pura ed applicata \\ Università degli Studi di Padova \\ Via G. Belzoni, 7 \\ 35131 PADOVA-ITALY \\ rampazzo@math.unipd.it
}

\section{Introduction}

In this note we present a simple application of the method developed by one of us in recent years to prove general smooth, nonsmooth, high-order, and hybrid versions of the maximum principle (abbr. MP) for finitedimensional, deterministic optimal control problems without state space constraints. As explained in Sussmann $[3,4,5,6]$, such versions can be derived in a unified way, by using a modified version of the approach of the classical book [2] by Pontryagin et al.. In the classical approach, one constructs "packets of needle variations," linearly approximates these packets at the base value of the variation parameter, and propagates the resulting linear approximations to the terminal point of the trajectory by means of the differentials of the reference flow maps. This technique must be modified by (a) replacing the classical differential by other objects, called generalized differentials (abbr. GDs), (b) replacing the time-varying vector fields that occur in the classical MP by flows, and (c) replacing the needle variations by abstract variations. A notion of GD will yield a version of the MP provided it satisfies some natural properties such as the chain rule and an appropriate "directional open mapping property." (Details are provided in $[3,4,5,6]$.)

Here we will show how to apply the general theory to derive a "nonsmooth" version of Chow's theorem, in which some of the vector fields involved are just continuous, while the others are Lipschitz continuous but not necessarily of class $C^{1}$. (We only consider the version involving brackets of two system vector fields. Versions involving higher-order brackets can be proved in a similar way, but require more regularity of the vector fields.) The result is the one to be expected, that is, that small-time local controllability from a point $x_{*}$ follows if the values at $x_{*}$ of the system vector fields, together with the Lie brackets at $x_{*}$ of the vector fields that are Lipschitz continuous, linearly span the whole space. To render this meaningful, one needs to give a precise definition of the notion of "Lie bracket" of two Lipschitz continuous vector fields $f, g$. This is done in $\S 5$, where we give a definition according to which $[f, g]\left(x_{*}\right)$ is always a nonempty compact convex set of vectors. Since the values of the Lie brackets are now sets, rather than single vectors, the notion of "linearly spanning the whole space" needs to be made precise. This is done in the statement of our main result, Theorem 2.1.

The GD theory used to prove Theorem 2.1 is that of "generalized differential quotients" (abbr. GDQs), so we include a section- $\$ 3$ - briefly reviewing the definition

*The second author was supported in part by NSF Grant DMS98-03411-00798. He is grateful to the Weizmann Institute Institute of Science, in Rehovot, Israel, where most of the work leading to this paper was done, while he was a Weston Visiting Professor during the Fall of 2000.

\author{
Héctor J. Sussmann \\ Department of Mathematics \\ Rutgers, the State University of New Jersey \\ Hill Center-Busch Campus \\ Piscataway, NJ 08854-8019, USA \\ sussmann@hamilton.rutgers.edu
}

and basic properties of this GD theory.

\section{Statement of the main theorem}

The result stated below involves the notion of "Lie bracket" $[f, g]$ of two locally Lipschitz vector fields $f$, $g$. In order to understand the statement, all the reader needs to know at this point is that for every $x$ belonging to the domain of $f$ and $g$ the bracket $[f, g](x)$ is a nonempty set of vectors, rather than a single vector as in the classical notion of Lie bracket of vector fields of class $C^{1}$. The precise definition of the Lie bracket of locally Lipschitz vector fields is given in $\S 5$ below, and is such that the set $[f, g](x)$ is always compact and convex.

Theorem 2.1 Assume that $n \in \mathbb{Z}, n \geq 0, \Omega$ is an open subset of $\mathbb{R}^{n}, f_{1}, \ldots, f_{m}, g_{1}, \ldots, g_{r}$ are vector fields on $\Omega, f_{1}, \ldots, f_{m}$ are continuous, and $g_{1}, \ldots, g_{r}$ are locally Lipschitz. Let $\Sigma$ be the driftless control system

$$
\Sigma: \quad \dot{x}=\sum_{i=1}^{m} u_{i} f_{i}(x)+\sum_{j=1}^{r} v_{j} g_{j}(x),
$$

with control constraints $\left|u_{i}\right| \leq 1$ for $i=1, \ldots, m$ and $\left|v_{j}\right| \leq 1$ for $j=1, \ldots, r$. Let $x_{*}$ be a point of $\Omega$ such that, for every choice $V=\left\{v_{k \ell}\right\}_{1 \leq k<\ell \leq r}$ of members $v_{k \ell} \in\left[g_{k}, g_{\ell}\right]\left(x_{*}\right)$ the set of vectors

$$
\begin{aligned}
& \left\{f_{i}(x): i=1, \ldots, m\right\} \cup\left\{g_{j}(x): j=1, \ldots, r\right\} \\
& \cup\left\{v_{k \ell}(x): k=1, \ldots, r-1, \ell=k+1, \ldots, r\right\}
\end{aligned}
$$

linearly spans $\mathbb{R}^{n}$. Then $\Sigma$ is locally controllable from $x_{*}$ in small time. More precisely, there exists a positive constant $A$ having the property that for every sufficiently small $\varepsilon$ it is possible to reach every point within a distance $\leq \varepsilon$ from $x_{*}$ in time not exceeding $A \sqrt{\varepsilon}$ by means of a piecewise constant bang-bang control such that at each time $t$ only one of the numbers $u_{1}(t), \ldots, u_{m}(t), v_{1}(t), \ldots, v_{r}(t)$ is nonzero.

\section{Generalized differential quotients (GDQs)}

A set-valued map (abbr. SVM) is a triple $F=(A, B, G)$ such that $A$ and $B$ are sets and $G$ is a subset of $A \times B$. The sets $A, B, G$ are, respectively, the source, target, and graph of $F$, and we write $A=\operatorname{So}(F)$, $B=\operatorname{Ta}(F), G=\operatorname{Gr}(F)$. If $x$ is any object, we write $F(x)=\{y:(x, y) \in \operatorname{Gr}(F)\}$. (Hence $F(x)=\emptyset$ unless $x \in \operatorname{So}(F)$.) The sets $\operatorname{Do}(F)=\{x \in \operatorname{So}(F): F(x) \neq \emptyset\}$, $\operatorname{Im}(F)=\bigcup_{x \in \operatorname{So}(F)} F(x)$, are, respectively, the domain and image of $F$. If $F=(A, B, G)$ is an SVM, we say that $F$ is an $S V M$ from $A$ to $B$, and write $F: A q \longmapsto B$. We use $S V M(A, B)$ to denote the set of all SVMs from $A$ to $B$.

If $X, Y$ are metric spaces, then $S V M_{\text {comp }}(X, Y)$ will denote the subset of $S V M(X, Y)$ whose members are the set-valued maps from $X$ to $Y$ that have a compact graph. We say that a sequence $\left\{F_{j}\right\}_{j \in \mathbb{N}}$ of 
members of $S V M_{\text {comp }}(X, Y)$ inward graph-converges to an $F \in S V M_{\text {comp }}(X, Y)$-and write $F_{j} \stackrel{\text { igr }}{\longrightarrow} F$-if for every open subset $\Omega$ of $X \times Y$ such that $\operatorname{Gr}(F) \subseteq \Omega$ there exists a $j_{\Omega} \in \mathbb{N}$ such that $\operatorname{Gr}\left(F_{j}\right) \subseteq \Omega$ whenever $j \geq j_{\Omega}$

Definition 3.1 Assume that $X, Y$ are metric spaces. A regular set-valued map from $X$ to $Y$ is a set-valued map $F \in S V M(X, Y)$ such that

- for every compact subset $K$ of $X$, the restriction $F\left[K\right.$ of $F$ to $K$ belongs to $S V M_{c o m p}(K, Y)$ and is a limit - in the sense of inward graph-convergenceof a sequence of continuous single-valued maps from $K$ to $Y$.

We use $\operatorname{REG}(X ; Y)$ to denote the set of all regular set-valued maps from $X$ to $Y$.

It is easy to see that if $F: X \mapsto Y$ is an ordinary (that is, single-valued and everywhere defined) map, then $F$ belongs to $\operatorname{REG}(X ; Y)$ if and only if $F$ is continuous.

It is not hard to prove the following.

Theorem 3.2 Assume that $X, Y, Z$ are metric spaces. Let $F \in \operatorname{REG}(X ; Y), G \in \operatorname{REG}(Y ; Z)$. Then the composite map $G \circ F$ belongs to $\operatorname{REG}(X ; Z)$.

Definition 3.3 Let $m, n \in \mathbb{Z}_{+}$, let $F: \mathbb{R}^{m} q \longmapsto \mathbb{R}^{n}$ be a set-valued map, and let $\Lambda$ be a nonempty compact subset of $\mathbb{R}^{n \times m}$. Let $S$ be a subset of $\mathbb{R}^{m}$. We say that $\Lambda$ is a generalized differential quotient (abbreviated "GDQ") of $F$ at $(0,0)$ in the direction of $S$, and write $\Lambda \in G D Q(F ; 0,0 ; S)$, if for every positive real number $\delta$ there exist $U, G$ such that

1. $U$ is a compact neighborhood of 0 in $\mathbb{R}^{m}$ and $U \cap S$ is compact;

2. $G$ is a regular set-valued map from $U \cap S$ to the $\delta$-neighborhood $\Lambda^{\delta}$ of $\Lambda$ in $\mathbb{R}^{n \times m}$;

3. $G(x) \cdot x \subseteq F(x)$ for every $x \in U \cap S$.

If $X, Y$ are real linear spaces, we use $\operatorname{Lin}(X, Y)$ to denote the set of all linear maps from $X$ to $Y$. A linear multimap from $X$ to $Y$ is a subset of $\operatorname{Lin}(X, Y)$. A linear multimultimap from $X$ to $Y$ is a set of linear multimaps from $X$ to $Y$.

If $M, N$ are $C^{1}$ manifolds, $\bar{x} \in M, \bar{y} \in N, S \subseteq M$, and $F: M q \mapsto N$, then we can define a linear multimultimap $G D Q(F ; \bar{x}, \bar{y} ; S)$ from $T_{\bar{x}} M$ to $T_{\bar{y}} N$ by picking coordinate charts $M \ni x \rightarrow \xi(x) \in \mathbb{R}^{m}$, $N \ni y \rightarrow \eta(y) \in \mathbb{R}^{n}$-where $m=\operatorname{dim} M, n=\operatorname{dim} N$ defined near $\bar{x}, \bar{y}$ and such that $\xi(\bar{x})=0, \eta(\bar{y})=0$, and declaring a subset $\Lambda$ of $\operatorname{Lin}\left(T_{\bar{x}} M, T_{\bar{y}} N\right)$ to belong to $G D Q(F ; \bar{x}, \bar{y} ; S)$ if the multimap $D \eta(\bar{y}) \circ \Lambda \circ D \xi(\bar{x})^{-1}$ is in $G D Q\left(\eta \circ F \circ \xi^{-1} ; 0,0 ; \xi(S)\right)$. It turns out that, with this definition, the set $G D Q(F ; \bar{x}, \bar{y} ; S)$ does not depend on the choice of the charts $\xi, \eta$. Moreover, the following four results can be proved.

Theorem 3.4 GDQ is a generalized differentiation theory in the sense of [4].

Theorem 3.5 GDQ has the strong directional open mapping property with linear rate (SDOMPLR).

The meaning of the SDOMPLR is as follows:
(SDOMPLR) Assume that $n, m$ are nonnegative integers, $F: \mathbb{R}^{n} q \mapsto \mathbb{R}^{m}, v \in \mathbb{R}^{m}, C$ is a closed convex cone in $\mathbb{R}^{n}, \Lambda$ belongs to $G D Q(F ; 0,0 ; C)$, and $v \in \bigcap_{L \in \Lambda} \operatorname{Int}(L C)$. Then there exist a closed convex cone $D$ in $\mathbb{R}^{n}$ and a function

$$
] 0,+\infty[\ni \delta \mapsto \varepsilon(\delta) \in] 0,+\infty[
$$

such that $v \in \operatorname{Int}(D)$, the function $\delta \mapsto \varepsilon(\delta)$ is linear for small enough $\delta$, and

$\left(^{*}\right)$ if $y \in D$ and $\|y\| \leq \varepsilon(\delta)$ then there exists a compact connected subset $\bar{Z}_{y}$ of $\{x \in C:\|x\| \leq \delta\} \times[0,1]$ such that $(0,0) \in Z_{y},(x, 1) \in Z_{y}$ for some $x \in C$ such that $\|x\| \leq \delta$, and $r y \in F(x)$ whenever $0 \leq r \leq 1$ and $(x, r) \in Z_{y}$.

Theorem 3.6 If $F: \mathbb{R}^{n} \mapsto \mathbb{R}^{m}$ is a continuous map, $x \in \mathbb{R}^{n}$, and $F$ is classically differentiable at $x$, then $\{D F(x)\} \in G D Q\left(F ; x, F(x) ; \mathbb{R}^{n}\right)$.

Theorem 3.7 If $F: \mathbb{R}^{n} \mapsto \mathbb{R}^{m}$ is Lipschitz-continuous, and $x \in \mathbb{R}^{n}$, then the Clarke generalized Jacobian $\partial F(x)$ belongs to $G D Q\left(F ; x, F(x) ; \mathbb{R}^{n}\right)$.

\section{Flows of continuous vector fields}

We assume throughout this section that

(A) $n \in \mathbb{Z}, n \geq 0$, and $\Omega$ is an open subset of $\mathbb{R}^{n}$.

If $f: \Omega \mapsto \mathbb{R}^{n}$ is a continuous vector field on $\Omega$, we use $\Phi^{f}$ to denote the flow of $f$. By definition, $\Phi^{f}$ is the setvalued map from $\Omega \times \mathbb{R}$ to $\Omega$ whose value, for a given $(x, t) \in \Omega \times \mathbb{R}$, is the set of all $y \in \Omega$ having the property that there exists an integral curve $\xi: I \rightarrow \Omega$ of $f$ which is defined on some subinterval $I$ of $\mathbb{R}$ such that $0 \in I$ and $t \in I$ and is such that $\xi(0)=x$ and $\xi(t)=y$. If $x_{*} \in \Omega$, we let $L^{f}\left(x_{*}\right)$ be the linear map from $\mathbb{R}^{n} \times \mathbb{R}$ to $\mathbb{R}^{n}$ given by

$$
L^{f}\left(x_{*}\right)(v, r)=v+r f\left(x_{*}\right) .
$$

Theorem 4.1 Assume (A) holds, and $f: \Omega \mapsto \mathbb{R}^{n}$ is a continuous vector field on $\Omega$. Let $x_{*} \in \Omega$. Then the set $\left\{L^{f}\left(x_{*}\right)\right\}$ is a generalized differential quotient of $\Phi^{f}$ at $\left(\left(x_{*}, 0\right), x_{*}\right)$ in the direction of $\mathbb{R}^{n}$.

Proof. If $v \in \mathbb{R}^{\nu}, w \in \mathbb{R}^{\mu}$, and $v \neq 0$, we let $E_{v, w}^{\nu, \mu}$ be the linear map from $\mathbb{R}^{\nu}$ to $\mathbb{R}^{\mu}$ given by

$$
E_{v, w}^{\nu, \mu}(u)=\frac{\langle v, u\rangle}{\|v\|^{2}} w \text { for } u \in \mathbb{R}^{\nu} .
$$

Then

$$
E_{v, w}^{\nu, \mu}(v)=w \quad \text { and } \quad\left\|E_{v, w}^{\nu, \mu}\right\|=\frac{\|w\|}{\|v\|} .
$$

Fix a positive number $\delta$. Let $\hat{\rho}$ be such that $\hat{\rho}>0$ and the compact ball $\hat{V}=\left\{x \in \mathbb{R}^{n}:\left\|x-x_{*}\right\| \leq \hat{\rho}\right\}$ is contained in $\Omega$. Let $\kappa=\sup \{\|f(x)\|: x \in \hat{V}\}$.

For every $\rho_{1}, \rho_{2}$ such that $0<\rho_{1} \leq \rho_{2} \leq \hat{\rho}$, let

$$
\begin{aligned}
& \omega\left(\rho_{1}, \rho_{2}\right) \stackrel{\text { def }}{=} \sup \left\{\left\|f(x)-f\left(x^{\prime}\right)\right\|:\right. \\
& \left.\left\|x-x^{\prime}\right\| \leq \rho_{1},\left\|x-x_{*}\right\| \leq \rho_{2},\left\|x^{\prime}-x_{*}\right\| \leq \rho_{2}\right\},
\end{aligned}
$$

so that

$$
\lim _{\rho_{1} \downarrow 0} \omega\left(\rho_{1}, \rho_{2}\right)=0 \text { if } 0<\rho_{2} \leq \hat{\rho} .
$$


Now fix $\bar{\rho}$ such that

$$
\bar{\rho}>0, \quad(2+\kappa) \bar{\rho} \leq \hat{\rho}, \quad \text { and } \quad \omega((1+\kappa) \bar{\rho}, \hat{\rho}) \leq \delta .
$$

Let $\bar{V}$ be the closed ball in $\mathbb{R}^{n}$ of radius $\bar{\rho}$, and center $x_{*}$, and let

$$
\bar{W}=\bar{V} \times[-\bar{\rho}, \bar{\rho}] .
$$

More generally, if $0<\rho \leq \bar{\rho}$, we let $V(\rho)$ be the closed ball in $\mathbb{R}^{n}$ of radius $\rho$ and center $x_{*}$, and then define $W(\rho)=V(\rho) \times[-\rho, \rho]$.

For $(x, t) \in \bar{W}, y \in \mathbb{R}^{n},(x, t) \neq\left(x_{*}, 0\right)$, define a linear $\operatorname{map} M_{x, t, y}: \mathbb{R}^{n} \times \mathbb{R} \mapsto \mathbb{R}^{n}$ by letting

$$
M_{x, t, y}=L^{f}\left(x_{*}\right)+E_{\left(x-x_{*}, t\right), y-x-t f\left(x_{*}\right)}^{n+1, n} .
$$

We then let $\Lambda(x, t)$ be the set of all linear maps $M_{x, t, y}$, for all $y \in \Phi^{f}(x, t)$.

We remark that if $t=0$ then $\Phi^{f}(x, t)=\{x\}$, so $\Lambda(x, t)=\left\{L^{f}\left(x_{*}\right)\right\}$. We extend the definition of $\Lambda$ by defining $\Lambda\left(x_{*}, 0\right)=\left\{L^{f}\left(x_{*}\right)\right\}$.

Now let $(x, t) \in \bar{W},(x, t) \neq\left(x_{*}, 0\right)$. If a linear map $M$ belongs to $\Lambda(x, t)$, and $M=M_{x, t, y}, y \in \Phi^{f}(x, t)$, then

$$
\begin{aligned}
x_{*}+M \cdot\left(x-x_{*}, t\right)= & x_{*}+L^{f}\left(x_{*}\right)\left(x-x_{*}, t\right) \\
& +\mathcal{E}_{x, t, y} \cdot\left(x-x_{*}, t\right) \\
= & x_{*}+x-x_{*}+t f\left(x_{*}\right) \\
& \quad+y-x-\operatorname{tf}\left(x_{*}\right) \\
= & y .
\end{aligned}
$$

where

$$
\mathcal{E}_{x, t, y} \stackrel{\text { def }}{=} E_{\left(x-x_{*}, t\right), y-x-t f\left(x_{*}\right)}^{n+1, n} .
$$

Therefore

$$
\Lambda(x, t) .\left(x-x_{*}, t\right) \subseteq \Phi^{f}(x, t) .
$$

Now suppose that $0<\rho \leq \bar{\rho},(x, t) \in W(\rho)$, and $M \in \Lambda(x, t)$. Write $M=M_{x, t, y}, y \in \Phi^{f}(x, t)$, and let $I_{t}$ denote the interval $[\min (0, t), \max (0, t)]$. Then

$$
\begin{aligned}
\left\|M_{x, t, y}-L^{f}\left(x_{*}\right)\right\| & =\left\|E_{\left(x-x_{*}, t\right), y-x-t f\left(x_{*}\right)}^{n+1, n}\right\| \\
& =\frac{\left\|y-x-t f\left(x_{*}\right) x\right\|}{\sqrt{\left\|x-x_{*}\right\|^{2}+t^{2}}} .
\end{aligned}
$$

But

$$
\left\|y-x-t f\left(x_{*}\right)\right\|=\left\|\int_{0}^{t}\left(f(\xi(s))-f\left(x_{*}\right)\right) d s\right\|,
$$

if $\xi: I_{t} \rightarrow \Omega$ is an integral curve of $f$ such that $\xi(0)=x$ and $\xi(t)=y$. Now, the curve $\xi$ satisfies $\dot{\xi}(s)=f(\xi(s))$, and then $\|\dot{\xi}(s)\| \leq \kappa$, whenever $\xi(s) \in \hat{V}$. Let $\tilde{I}$ be the set of all $s \in I_{t}$ such that $\xi(\sigma) \in \hat{V}$ for all $\sigma \in I_{s}$. Then $\tilde{I}$ is a compact subinterval of $I_{t}$ such that $0 \in I_{s}$. So $\tilde{I}=I_{\tau}$ for some $\tau \in I_{t}$. If $\tau \neq t$ then $\left\|\xi(\tau)-x_{*}\right\|=\hat{\rho}$. But $\|\xi(s)-x\| \leq \kappa|s|$ for all $s \in \tilde{I}$, and then the inequality $\left\|\xi(\tau)-x_{*}\right\| \leq \kappa|s|+\rho$ holds for all such $s$. Therefore $\left\|\xi(\tau)-x_{*}\right\| \leq(1+\kappa) \rho \leq(1+\kappa) \bar{\rho}<\hat{\rho}$ contradicting the fact that $\left\|\xi(\tau)-x_{*}\right\|=\hat{\rho}$. It follows that $\tau=t$ and $\tilde{I}=I_{t}$, so that

$$
\left\|\xi(s)-x_{*}\right\| \leq(1+\kappa) \rho \quad \text { whenever } \quad s \in I_{t} .
$$

Then

$$
\left\|f(\xi(s))-f\left(x_{*}\right)\right\| \leq \omega((1+\kappa) \rho, \hat{\rho}) \text { if } s \in I_{t}, .
$$

Therefore

$$
\left\|y-x-t f\left(x_{*}\right)\right\| \leq|t| \omega((1+\kappa) \rho, \hat{\rho}) .
$$

If we pick $\rho=\bar{\rho}$, we get

from which it follows that

$$
\left\|y-x-t f\left(x_{*}\right)\right\| \leq|t| \delta
$$

$$
\frac{\left\|y-x-t f\left(x_{*}\right)\right\|}{\sqrt{\left\|x-x_{*}\right\|^{2}+t^{2}}} \leq \delta,
$$

and then $\left\|M_{x, t, y}-L^{f}\left(x_{*}\right)\right\| \leq \delta$.

So we have shown that

$$
\left\|M-L^{f}\left(x_{*}\right)\right\| \leq \delta \quad \text { whenever } \quad M \in \Lambda(x, t),
$$

as long as $(x, t) \in \bar{W}$ and $(x, t) \neq\left(x_{*}, 0\right)$.

On the other hand, given any $(x, t) \in \bar{W}$, we can pick $\rho=\max \left(\left\|x-x_{*}\right\|,|t|\right)$, and then get the estimate

$$
\begin{gathered}
\left\|M-L^{f}\left(x_{*}\right)\right\| \leq \omega\left(\max \left(\left\|x-x_{*}\right\|,|t|\right), \hat{\rho}\right) \\
\text { whenever } \quad(x, t) \in \bar{W}, M \in \Lambda(x, t),
\end{gathered}
$$

It is clear that equations (3) and (5), which have been proved under the assumption that $(x, t) \neq\left(x_{*}, 0\right)$, are also valid when $(x, t)=\left(x_{*}, 0\right)$. So $(3)$ and $(5)$ hold for all $(x, t) \in \bar{W}$.

To complete our proof, we have to show that the setvalued map $\bar{W} \ni(x, t) \mapsto \Lambda(x, t) \subseteq \operatorname{Lin}\left(\mathbb{R}^{n+1}, \mathbb{R}^{n}\right)$ is regular. To prove this, we first have to prove that the set

$$
G=\{(x, t, M):(x, t) \in \bar{W}, M \in \Lambda(x, t)\}
$$

is compact.

For this purpose, we let $\left\{\left(x^{j}, t^{j}, M^{j}\right)\right\}_{j \in \mathbb{N}}$ be a sequence of members of $G$, and try to extract a subsequence that converges to a member of $G$.

If $\left(x^{j}, t^{j}\right)=\left(x_{*}, 0\right)$ for infinitely many indices $j$, then $M=L^{f}\left(x_{*}\right)$ for all such indices, so the existence of the desired subsequence is obvious. Otherwise, we may assume that $\left(x^{j}, t^{j}\right) \neq\left(x_{*}, 0\right)$ for all indices $j$. Write $M^{j}=M_{x^{j}, t^{j}, y^{j}}$, where $y^{j} \in \Phi^{f}\left(x^{j}, t^{j}\right)$. We may clearly assume that the sequence $\left\{x^{j}, t^{j}\right\}_{j \in \mathbb{N}}$ itself converges to a limit $(\bar{x}, \bar{t})$. Now choose integral curves $\xi^{j}$ of $f$, defined on the intervals $I^{j}=I_{t^{j}}$, such that $\xi^{j}(0)=x^{j}$ and $\xi^{j}\left(t^{j}\right)=y^{j}$. As in the proof of (4), we conclude that $\xi^{j}(s) \in \hat{V}$ whenever $s \in I^{j}$. Then $\left\|\dot{\xi}^{j}(s)\right\|=\left\|f\left(\xi^{j}(s)\right)\right\| \leq \kappa$, so the sequence $\left\{\xi^{j}\right\}_{j \in \mathbb{N}}$ is equicontinuous. Since this sequence is also uniformly bounded, we may assume, by passing to a subsequence, that the curves $\xi^{j}$ converge uniformly to a limit curve $\xi$, defined on $I_{t}$. It then follows by familiar arguments that $\bar{y}=\xi(t) \in \Phi^{f}(\bar{x}, \bar{t})$.

Let $\bar{M}=M_{\bar{x}, \bar{t}, \bar{y}}$ if $(\bar{x}, \bar{t}) \neq\left(x_{*}, 0\right), \bar{M}=L^{f}\left(x_{*}\right)$ if $(\bar{x}, \bar{t})=\left(x_{*}, 0\right)$. Then $\bar{M} \in \Lambda(\bar{x}, \bar{t})$. Let us prove that $M^{j} \rightarrow \bar{M}$ as $j \rightarrow \infty$. Assume first that $(\bar{x}, \bar{t}) \neq\left(x_{*}, 0\right)$. Since $x^{j} \rightarrow \bar{x}, t^{j} \rightarrow \bar{t}$, and $y^{j} \rightarrow \bar{y}$, if follows from (2) that $M^{j} \rightarrow \bar{M}$, since the maps $(v, w) \mapsto E_{v, w}^{\nu, \mu}$ are continuous on $\left(\mathbb{R}^{\nu} \backslash\{0\}\right) \times \mathbb{R}^{\mu}$. Now assume that $(\bar{x}, \bar{t})=\left(x_{*}, 0\right)$. Then $x^{j} \rightarrow x_{*}$ and $t^{j} \rightarrow 0$. So $(6)$ implies that $M^{j} \rightarrow L^{f}\left(x_{*}\right)=\bar{M}$.

We must now show that $\Lambda$ is a limit, in the sense of inward graph convergence of set-valued maps from $\bar{W}$ to $\operatorname{Lin}\left(\mathbb{R}^{n+1}, \mathbb{R}^{n}\right)$, of single-valued continuous maps. To prove this, we first let $F$ be a continuous map from $\mathbb{R}^{n}$ to $\mathbb{R}^{n}$ which coincides with $f$ on $\hat{V}$ and is such that $\|F(x)\| \leq \kappa$ for all $x \in \mathbb{R}^{n}$. We then let $F^{\zeta}$ be the usual regularizations of $F$, defined-for $\zeta>0$-by letting

$$
F^{\zeta}(x)=\int_{\mathbb{R}^{n}} \varphi(h) F(x+\zeta h) d h,
$$


where $\varphi$ is a nonnegative real-valued function on $\mathbb{R}^{n}$ of class $C^{\infty}$ such that $\int_{\mathbb{R}^{n}} \varphi=1$ and $\varphi(h)=0$ whenever $\|h\|>1$. .

Then $F^{\zeta} \in C^{\infty}$ for all positive $\zeta,\left\|F^{\zeta}(x)\right\| \leq \kappa$ for all $x \in \mathbb{R}^{n}$ and all positive $\zeta$, and $F^{\zeta} \rightarrow F$ uniformly on compact sets as $\zeta \downarrow 0$. The flow maps $\Phi^{F^{\zeta}}$ are then globally defined, single-valued, and smooth. We construct maps $\Lambda^{\zeta}$, defined on $\bar{W}$, exactly as we constructed $\Lambda$, using $F^{\zeta}$ instead of $f$. Then the $\Lambda^{\zeta}$ are single-valued and continuous. Moreover, the moduli of continuity

$$
\begin{aligned}
& \omega^{\zeta}\left(\rho_{1}, \rho_{2}\right) \stackrel{\text { def }}{=} \sup \left\{\left\|F^{\zeta}(x)-F^{\zeta}\left(x^{\prime}\right)\right\|:\right. \\
& \left.\left\|x-x^{\prime}\right\| \leq \rho_{1},\left\|x-x_{*}\right\| \leq \rho_{2},\left\|x^{\prime}-x_{*}\right\| \leq \rho_{2}\right\}
\end{aligned}
$$

of the $F^{\zeta}$ are related to the modulus of continuity $\omega$ of $f$ by

$$
\omega^{\zeta}\left(\rho_{1}, \rho_{2}\right) \leq \omega\left(\rho_{1}, \rho_{2}+\zeta\right) .
$$

Let us show that $\Lambda^{\zeta} \stackrel{\text { igr }}{\longrightarrow} \Lambda$. Let

$$
\alpha(\zeta)=\sup \left\{d(q, G): q \in G^{\zeta}\right\},
$$

where

$$
G^{\zeta}=\left\{(x, t, M):(x, t) \in \bar{W}, M \in \Lambda^{\zeta}(x, t)\right\} .
$$

We want to prove that $\alpha(\zeta) \rightarrow 0$ as $\zeta \downarrow 0$. Suppose this is not true. Then there exist a sequence $\left\{\zeta_{j}\right\}_{j \in \mathbb{N}}$ of positive numbers and an $\bar{\alpha}$ such that $\zeta_{j} \downarrow 0, \bar{\alpha}>0$, and $\alpha\left(\zeta_{j}\right) \geq 2 \bar{\alpha}$. Write $G_{j}=G^{\zeta_{j}}$. Pick $q_{j} \in G_{j}$ such that $d\left(q_{j}, G\right) \geq \bar{\alpha}$. Write $q_{j}=\left(x_{j}, t_{j}, M_{j}\right)$. We will reach a contradiction by finding a subsequence of $\left\{q_{j}\right\}_{j \in \mathbb{N}}$ such that $d\left(q_{j}, G\right) \rightarrow 0$. For this purpose, we may assume, after passing to a subsequence, that $\left(x_{j}, t_{j}\right)$ converges to a limit $(x, t) \in \bar{W}$.

Let $J$ be the set of all indices $j$ such that $\left(x_{j}, t_{j}\right)=\left(x_{*}, 0\right)$. If $J$ is infinite, then $M_{j}=L^{F^{\zeta_{j}}}\left(x_{*}\right)$ for $j \in J$, so $\lim _{j \rightarrow \infty, j \in J} M_{j}=L^{f}\left(x_{*}\right)$. Then $\lim _{j \rightarrow \infty, j \in J} q_{j}=\left(x_{*}, 0, L^{f}\left(x_{*}\right)\right) \in G$ so $\lim _{j \rightarrow \infty, j \in J} d\left(q_{j}, G\right)=0$, showing the existence of the desired subsequence.

Next, let us assume that $J$ is finite. Then, by passing to a subsequence, we may assume that $J=\emptyset$, i.e., that $\left(x_{j}, t_{j}\right) \neq\left(x_{*}, 0\right)$ for all $j$. Let $y_{j}=\Phi^{F^{\zeta_{j}}}\left(x_{j}, t_{j}\right)$. Then

$$
M_{j}=L^{F^{\zeta_{j}}}\left(x_{*}\right)+E_{\left(x_{j}-x_{*}, t_{j}\right), y_{j}-x_{j}-t_{j} F^{\zeta_{j}}\left(x_{*}\right)}^{n+1, n} .
$$

For each $j$, find an integral curve $\xi_{j}: I_{t_{j}} \mapsto \mathbb{R}^{n}$ of $F^{\zeta_{j}}$ such that $\xi_{j}(0)=t_{j}$ and $\xi_{j}\left(t_{j}\right)=y_{j}$. Then $\left\|\dot{\xi}_{j}(s)\right\| \leq \kappa$ for all $j$, so $\xi_{j}$ is entirely contained in $\hat{V}$, and then the sequence $\left\{\xi_{j}\right\}_{j \in \mathbb{N}}$ is bounded and equicontinuous. By passing to a subsequence, we may assume that $\xi_{j} \rightarrow \xi$ uniformly, for some curve $\xi: I_{t} \rightarrow \mathbb{R}^{n}$. Since $\xi_{j}(0)=x_{j}$ and $\xi_{j}\left(t_{j}\right)=y_{j}$, it is clear that $\xi(0)=x$ and $\xi(t)=y=\lim _{j \rightarrow \infty} y_{j}$. Moreover, $\xi$ is an integral curve of $f$, so $y \in \Phi^{f}(x, t)$.

Now assume that $(x, t) \neq\left(x_{*}, 0\right)$. Then, using the facts that (a) the maps $(v, w) \mapsto E_{v, w}^{\nu, \mu}$ are continuous on $\left(\mathbb{R}^{\nu} \backslash\{0\}\right) \times \mathbb{R}^{\mu}$ and (b) $L^{F^{\zeta_{j}}}\left(x_{*}\right) \rightarrow L^{f}\left(x_{*}\right)$, we can conclude from (9) that $M_{j} \rightarrow M$, where $M=L^{f}\left(x_{*}\right)+E_{\left(x-x_{*}, t\right), y-x-t f\left(x_{*}\right)}^{n+1, n}$. But this tells us, using the definition of $\Lambda$, that $M \in \Lambda(x, t)$. Therefore $q=(x, t, M) \in G$. Since $q_{j} \rightarrow q$, we see that $d\left(q_{j}, G\right) \rightarrow 0$, as desired.
Finally, we have to consider the case when $(x, t)=\left(x_{*}, 0\right)$. In that case, we use the estimate

$$
\begin{gathered}
\left\|M-L^{F^{\zeta}}\left(x_{*}\right)\right\| \leq \omega^{\zeta}\left(\max \left(\left\|x-x_{*}\right\|,|t|\right), \hat{\rho}\right) \\
\text { whenever } \quad(x, t) \in \bar{W}, M \in \Lambda^{\zeta}(x, t), \quad
\end{gathered}
$$

which is proved in the same way as (6). In view of (8), we have

$$
\left\|M_{j}-L^{F^{\zeta_{j}}}\left(x_{*}\right)\right\| \leq \omega\left(\max \left(\left\|x_{j}-x_{*}\right\|,\left|t_{j}\right|\right), \hat{\rho}+\beta\right)
$$

for sufficiently large $j$, if $\beta$ is a fixed positive number. Therefore $\left\|M_{j}-L^{F^{\zeta_{j}}}\left(x_{*}\right)\right\| \rightarrow 0$. Since $L^{F^{\zeta_{j}}}\left(x_{*}\right) \rightarrow L^{f}\left(x_{*}\right)$, we can conclude that $M_{j} \rightarrow L^{f}\left(x_{*}\right)$. Now, by the definition of $\Lambda$, $\left.L^{f}\left(x_{*}\right)\right) \in \Lambda\left(x_{*}, t\right)$. So, if we let $q=\left(x_{*}, 0, L^{f}\left(x_{*}\right)\right)$, then $q \in G$. Since $q_{j} \rightarrow q$, we see that $d\left(q_{j}, G\right) \rightarrow 0$ in this case as well, and our proof is complete.

\section{Lie brackets of locally Lipschitz vector fields}

We assume throughout this section that (A) holds. If $f$ is a locally Lipschitz vector field on $\Omega$, we let $D I F F(f)$ denote the set of points $x \in \Omega$ such that $f$ is differentiable at $x$. Then Rademacher's theorem implies that $D I F F(f)$ is a subset of full measure of $\Omega$. If $x \in D I F F(f)$, then $D f(x)$ will denote the differential of $f$ at $x$.

Now let $f, g$ be two locally Lipschitz vector fields on $\mathbb{R}^{n}$, and let $x \in \Omega$. We define $[f, g](x)$ - the Lie bracket of $f$ and $g$ at $x$-to be the convex hull of the set of all vectors

$$
v=\lim _{j \rightarrow \infty}\left(D g\left(x_{j}\right) \cdot f\left(x_{j}\right)-D f\left(x_{j}\right) \cdot g\left(x_{j}\right)\right),
$$

for all sequences $\left\{x_{j}\right\}_{j \in \mathbb{N}}$ such that

1. $x_{j} \in D I F F(f) \cap D I F F(g)$ for all $j$,

2. $\lim _{j \rightarrow \infty} x_{j}=x$,

3. the limit $v$ of (12) exists.

It is clear that the set $[f, g](x)$ is convex, compact, and nonempty for every $x \in \Omega$. Moreover, the skewsymmetry identity

clearly holds for all $x$.

$$
[f, f](x)=\{0\}
$$

If $f$ is a locally Lipschitz vector field, $x \in \Omega$, and $t \in \mathbb{R}$, we use $x e^{t f}$ to denote the value at time $t$ of the integral curve $\xi$ of $f$ such that $\xi(0)=x$. (That is, $x e^{t f}$ is the unique member of $\Phi^{f}(x, t)$.)

Given two locally Lipschitz vector fields, $x \in \Omega$, and $t, s \in \mathbb{R}$, we define

$$
\Psi^{f, g}(x, t, s)=x e^{t f} e^{s g} e^{-t f} e^{-s g} .
$$

It is clear that if $K$ is an arbitrary compact subset of $\Omega$, then there exists a positive time $\tau_{K}$ such that $\Psi^{f, g}(x, t, s)$ is defined whenever $x \in K,|t| \leq \tau_{K}$ and $|s| \leq \tau_{K}$.

We now derive an integral formula for $\Psi^{f, g}(x, t, s)-x$ when $f$ and $g$ are smooth. Formally,

$$
\begin{aligned}
& \Psi^{f, g}(x, t, s)-x \\
= & x e^{t f} e^{s g} e^{-t f} e^{-s g}-x \\
= & \int_{0}^{s}\left(x e^{t f} e^{\sigma g} g e^{-t f} e^{-\sigma g}-x e^{t f} e^{\sigma g} e^{-t f} g e^{-\sigma g}\right) d \sigma
\end{aligned}
$$




$$
\begin{aligned}
& =\int_{0}^{s}\left(x e^{t f} e^{\sigma g} e^{-t f}\left(e^{t f} g e^{-t f}-g\right) e^{-\sigma g}\right) d \sigma \\
& =\int_{0}^{s} \int_{0}^{t}\left(x e^{t f} e^{\sigma g} e^{-t f}\left(e^{\tau f}[f, g] e^{-\tau f}\right) e^{-\sigma g}\right) d \tau d \sigma \\
& =\int_{0}^{s} \int_{0}^{t}\left(x e^{t f} e^{\sigma g} e^{(\tau-t) f}[f, g] e^{-\tau f} e^{-\sigma g}\right) d \tau d \sigma .
\end{aligned}
$$

The above formula can be given a precise interpretation and a rigorous proof, by regarding all the expressions involved as distributions (cf. for example [1]).

For our purposes, what we need to know about the integral formula for $\Psi^{f, g}(x, t, s)-x$ is that the term $x e^{t f} e^{\sigma g} e^{(\tau-t) f}[f, g] e^{-\tau f} e^{-\sigma g}$ is the vector determined as follows: we compute the Lie bracket $[f, g]$ of $f$ and $g$ at the point

$$
\theta(x, \sigma, t, \tau) \stackrel{\text { def }}{=} x e^{t f} e^{\sigma g} e^{(\tau-t) f}
$$

and transport this vector to the point

$$
\begin{aligned}
\Psi^{f, g}(x, t, \sigma) & =x e^{t f} e^{\sigma g} e^{-t f} e^{-\sigma g} \\
& =\theta(x, \sigma, t, \tau) e^{-\tau f} e^{-\sigma g}
\end{aligned}
$$

by means of the differential at $\theta(x, \sigma, t, \tau)$ of the diffeomorphism $y \mapsto y e^{-\tau f} e^{-\sigma g}$.

If $X$ is a smooth vector field, then to transport a tangent vector $\bar{v}$ from a point $\bar{y}$ to the point $\bar{y} e^{\bar{t} X}$ by means of the differential of the map $y \mapsto y e^{\bar{t} X}$, one solves the variational equation

$$
\dot{v}(t)=D X\left(y e^{t X}\right) \cdot v(t)
$$

with initial condition $v(0)=\bar{v}$. The desired vector is then $v(\bar{t})$. If $C$ is a constant such that $\|D X(x)\| \leq C$ for all $x$, then Gronwall's inequality implies the bound

$$
\|v(t)\| \leq e^{|t| C}\|\bar{v}\|,
$$

from which it follows that $\|v(t)-\bar{v}\| \leq C|t| e^{|t| C}\|\bar{v}\|$. Therefore

$$
\|v(r)-\bar{v}\| \leq C|r| e^{|r| C}\|\bar{v}\| .
$$

If we apply this to the integrand $x e^{t f} e^{\sigma g} e^{(\tau-t) f}[f, g] e^{-\tau f} e^{-\sigma g}$, we conclude that

$$
\begin{gathered}
\left\|x e^{t f} e^{\sigma g} e^{(\tau-t) f}[f, g] e^{-\tau f} e^{-\sigma g}-[f, g](\theta(x, \sigma, t, \tau))\right\| \\
\leq C|s| e^{|s| C}\left(1+C|t| e^{|t| C}\right)\|[f, g](\theta(x, \sigma, t, \tau))\|,
\end{gathered}
$$

from which we get the estimate

$$
\begin{gathered}
\left\|\Psi^{f, g}(x, t, s)-x-\int_{0}^{s} \int_{0}^{t}([f, g](\theta(x, \sigma, t, \tau))) d \tau d \sigma\right\| \\
\leq 2 C^{3}|s|^{2}|t| e^{|s| C}\left(1+C|t| e^{|t| C}\right),
\end{gathered}
$$

where $C$ is an upper bound for $\|f(y)\|,\|D f(y)\|,\|g(y)\|$, and $\|D g(y)\|$ for all $y$.

Now suppose that $f$ and $g$ are just Lipschitz. Fix a point $x_{*} \in \Omega$. Fix a positive number $\hat{\rho}$ such that the compact ball

$$
\hat{V}=\left\{x \in \mathbb{R}^{n}:\left\|x-x_{*}\right\| \leq \bar{\rho}\right\}
$$

is contained in $\Omega$. Let $C_{1}$ be an upper bound for $\|f(x)\|$ and $\|g(x)\|$ for all $x \in \hat{V}$, and let $C_{2}$ be a Lipschitz constant for $f$ and $g$ on $\hat{V}$. Then let $C=\max \left(C_{1}, C_{2}\right)$.

For $0<\rho \leq \hat{\rho}$, use $\gamma(\rho)$ to denote the supremum of the distances $d\left(D g(x) \cdot f(x)-D f(x) \cdot g(x),[f, g]\left(x_{*}\right)\right)$, for $x \in D I F F(f) \cap D I F F(g)$ such that $\left\|x-x_{*}\right\| \leq \rho$.
It is then easy to see that

$$
\lim _{\rho \downarrow 0} \gamma(\rho)=0 .
$$

Now let $f^{\zeta}, g^{\zeta}$ be the regularizations of $f, g$, defined as before for positive $\zeta$. Then, after replacing $\hat{V}$ by a smaller ball, and as long as $\zeta$ is sufficiently small, the constant $C$ is also an upper bound for $\left\|f^{\zeta}(x)\right\|$ and $\left\|g^{\zeta}(x)\right\|$ for all $x$ and a Lipschitz constant for $f$ and $g$. Then we can apply (14) to the regularized vector fields, and conclude that

$$
\begin{aligned}
& \| \Psi^{f^{\zeta}, g^{\zeta}}(x, t, s)-x \\
& -\int_{0}^{s} \int_{0}^{t}\left(\left[f^{\zeta}, g^{\zeta}\right]\left(\theta^{\zeta}(x, \sigma, t, \tau)\right)\right) d \tau d \sigma \| \\
& \quad \leq 2 C^{3}|s|^{2}|t| e^{|s| C}\left(1+C|t| e^{|t| C}\right)
\end{aligned}
$$

if $|s|,|t|$ and $\zeta$ are small enough, where $\theta^{\zeta}$ is defined as in (13), using $f^{\zeta}$ and $g^{\zeta}$ instead of $f$ and $g$.

For any given $y$, we would like to estimate the distance

We write

$$
\Delta(y, \zeta)=d\left(\left[f^{\zeta}, g^{\zeta}\right](y),[f, g]\left(x_{*}\right)\right) .
$$

$$
\begin{aligned}
& {\left[f^{\zeta}, g^{\zeta}\right](y)=D g^{\zeta}(y) \cdot f^{\zeta}(y)-D f^{\zeta}(y) \cdot g^{\zeta}(y) } \\
= & \int_{\mathbb{R}^{n}} \varphi(h) D g(y+\zeta h) \cdot f^{\zeta}(y) d h \\
& \quad-\int_{\mathbb{R}^{n}} \varphi(h) D f(y+\zeta h) \cdot g^{\zeta}(y) d h \\
= & I_{1}(t)+I_{2}(y),
\end{aligned}
$$

where

$$
\begin{aligned}
I_{1}(y)= & \int_{\mathbb{R}^{n}} \varphi(h) w(y+\zeta h) d h, \\
w(z)= & D g(z) \cdot f(z)-D f(z) \cdot g(z), \\
I_{2}(y)= & \int_{\mathbb{R}^{n}} \varphi(h) D g(y+\zeta h) \cdot\left(f^{\zeta}(y)-f(y+\zeta h)\right) d h \\
& -\int_{\mathbb{R}^{n}} \varphi(h) D f(y+\zeta h) \cdot\left(g^{\zeta}(y)-g(y+\zeta h)\right) d h .
\end{aligned}
$$

The integral $I_{1}(y)$ is an average of vectors of the form $D g(z) \cdot f(z)-D f(z) \cdot g(z)$, for points $z=y+\zeta h$ such that $\left\|z-x_{*}\right\| \leq\left\|y-x_{*}\right\|+\zeta$. Therefore

$$
d\left(I_{1}(y),[f, g]\left(x_{*}\right)\right) \leq \gamma\left(\left\|y-x_{*}\right\|+\zeta\right) .
$$

On the other hand, it is clear that $\left\|f^{\zeta}(y)-f(y)\right\| \leq C \zeta$ for every $y$, so

$$
\begin{aligned}
& \left\|f^{\zeta}(y)-f(y+\zeta h)\right\| \leq\left\|f^{\zeta}(y)-f(y)\right\|+\|f(y)-f(y+\zeta h)\| \\
& \quad \leq 2 C \zeta,
\end{aligned}
$$

and then $\left\|I_{2}(y)\right\| \leq 4 C^{2} \zeta$. It follows that

$$
\Delta(y, \zeta) \leq \gamma\left(\left\|y-x_{*}\right\|+\zeta\right)+4 C^{2} \zeta .
$$

Now, if $y=\theta^{\zeta}(x, \sigma, t, \tau)$, where, as before, $\sigma \in I_{s}$ and $\tau \in I_{t}$, then $\|y-x\| \leq C(2|t|+|s|)$, so

$$
\left.\left\|y-x_{*}\right\| \leq C(2|t|+|s|)+\left\|x-x_{*}\right\|\right),
$$

and then the distance from $\left[f^{\zeta}, g^{\zeta}\right](y)$ to $[f, g]\left(x_{*}\right)$ is bounded by $\left.\gamma\left(C(2|t|+|s|)+\left\|x-x_{*}\right\|\right)+\zeta\right)+4 C^{2} \zeta$.

If $s t>0$, it follows that

$$
\begin{aligned}
& d\left((t s)^{-1}\left(\Psi^{f^{\zeta}, g^{\zeta}}(x, t, s)-x\right),[f, g]\left(x_{*}\right)\right) \\
& \leq 2 C^{3}|s| e^{|s| C}\left(1+C|t| e^{|t| C}\right) \\
& +\gamma\left(C(2|t|+|s|)+\left\|x-x_{*}\right\|+\zeta\right)+4 C^{2} \zeta .
\end{aligned}
$$


We can now let $\zeta \downarrow 0$, and conclude that

$$
\begin{aligned}
& d\left((t s)^{-1}\left(\Psi^{f, g}(x, t, s)-x\right),[f, g]\left(x_{*}\right)\right) \\
& \leq 2 C^{3}|s| e^{|s| C}\left(1+C|t| e^{|t| C}\right)+\gamma(C(2|t|+|s|)) \\
& \leq \Gamma(\max (|t|,|s|), x)
\end{aligned}
$$

where

$$
\Gamma(r, x)=2 C^{3} r e^{r C}\left(1+C r e^{r C}\right)+\gamma\left(3 r C+\left\|x-x_{*}\right\|\right) .
$$

We now define a map $(x, \varepsilon) \mapsto \Xi^{f, g}(x, \varepsilon)$ by letting

$$
\Xi^{f, g}(x, \varepsilon)= \begin{cases}\Psi^{f, g}(x, \sqrt{\varepsilon}, \sqrt{\varepsilon}) & \text { if } \quad \varepsilon \geq 0, \\ \Psi^{f,-g}(x, \sqrt{-\varepsilon}, \sqrt{-\varepsilon}) & \text { if } \quad \varepsilon \leq 0 .\end{cases}
$$

Then $\Xi^{f, g}$ is continuous. (Notice that when $\varepsilon=0$ the vectors $\Psi^{f, g}(x, \sqrt{\varepsilon}, \sqrt{\varepsilon})$ and $\Psi^{f,-g}(x, \sqrt{-\varepsilon}, \sqrt{-\varepsilon})$ are both equal to $x$.) Moreover, (16) implies the inequalities

$$
\begin{aligned}
d\left(\varepsilon^{-1}\left(\Xi^{f, g}(x, \varepsilon)-x\right),[f, g]\left(x_{*}\right)\right) & \leq \Gamma(\sqrt{\varepsilon}, x), \\
d\left(\frac{\Xi^{f, g}(x, \varepsilon)-x}{-\varepsilon},[f,-g]\left(x_{*}\right)\right) & \leq \Gamma(\sqrt{-\varepsilon}, x)
\end{aligned}
$$

valid, respectively, when $\varepsilon>0$ and $\varepsilon<0$. Since $[f,-g]\left(x_{*}\right)=-[f, g]\left(x_{*}\right),(18)$ is equivalent to

$$
d\left(\varepsilon^{-1}\left(\Xi^{f, g}(x, \varepsilon)-x\right),[f, g]\left(x_{*}\right)\right) \leq \Gamma(\sqrt{-\varepsilon}, x) .
$$

We have therefore proved that the inequality

$$
d\left(\varepsilon^{-1}\left(\Xi^{f, g}(x, \varepsilon)-x\right),[f, g]\left(x_{*}\right)\right) \leq \Gamma(\sqrt{|\varepsilon|}, x)
$$

is valid for all sufficiently small $\varepsilon$, positive or negative. Equivalently, we have shown that

$$
\begin{aligned}
& \inf \left\{\left\|\Xi^{f, g}(x, \varepsilon)-x-\varepsilon v\right\|: v \in[f, g]\left(x_{*}\right)\right\} \\
& \quad=o\left(\varepsilon+\left\|x-x_{*}\right\|\right) .
\end{aligned}
$$

For a given $w \in \mathbb{R}^{n}$, let $Q^{w}$ denote the linear map

$$
\mathbb{R}^{n+1} \sim \mathbb{R}^{n} \times \mathbb{R} \ni(v, r) \mapsto v+r w \stackrel{\text { def }}{=} Q^{w}(v, r) \in \mathbb{R}^{n} .
$$

If $W$ is a subset of $\mathbb{R}^{n}$, write $\mathcal{Q}^{W} \stackrel{\text { def }}{=}\left\{Q^{w}: w \in W\right\}$.

Then (21) says - since $\Xi^{f, g}\left(x_{*}, 0\right)=x_{*}$ - that

$$
\begin{aligned}
& \inf \left\{\left\|\Xi^{f, g}(x, \varepsilon)-\Xi^{f, g}\left(x_{*}, 0\right)-Q^{v}\left(x-x_{*}, \varepsilon\right)\right\|\right. \\
& \left.\quad: v \in[f, g]\left(x_{*}\right)\right\}=o\left(\varepsilon+\left\|x-x_{*}\right\|\right),
\end{aligned}
$$

i.e., that

$$
\begin{aligned}
& \inf \left\{\left\|\Xi^{f, g}(x, \varepsilon)-\Xi^{f, g}\left(x_{*}, 0\right)-M\left(x-x_{*}, \varepsilon\right)\right\|\right. \\
& \left.\quad: M \in \mathcal{Q}^{[f, g]\left(x_{*}\right)}\right\}=o\left(\varepsilon+\left\|x-x_{*}\right\|\right) .
\end{aligned}
$$

The following result then follows from the general sufficient condition given in [5] for a set to be a GDQ:

Theorem 5.1 Assume (A) holds. Let $f, g$ be locally Lipschitz vector fields on $\Omega$. Let $x_{*} \in \Omega$. Then the set $\mathcal{Q}^{[f, g]\left(x_{*}\right)}$ is a generalized differential quotient of the map $\Xi^{f, g}$ at $\left(x_{*}, 0\right)$ in the direction of $\mathbb{R}^{n}$.

\section{Proof of the main theorem}

First, we make a slight change of notation, and write

$$
\Phi^{f, \varepsilon}(x)=\Phi^{f}(x, \varepsilon), \quad \Xi^{f, g, \varepsilon}(x)=\Xi^{f, g}(x, \varepsilon) .
$$

Let $S=\{(i, j) \in \mathbb{Z} \times \mathbb{Z}: 1 \leq i<j \leq r\}, s=\frac{r(r-1)}{2}$, so $\operatorname{card}(S)=s$. Fix a bijection $\{1, \ldots, s\} \ni k \mapsto\left(i_{k}, j_{k}\right) \in S$ from $\{1, \ldots, s\}$ onto $S$. Let $X=\mathbb{R}^{m+r+s} \sim \mathbb{R}^{m} \times \mathbb{R}^{r} \times \mathbb{R}^{s}$. Define a map $\Theta$ from a neighborhood of 0 in $X$ to $\mathbb{R}^{n}$, by letting

$$
\begin{aligned}
& \Theta\left(\varepsilon_{1}, \ldots, \varepsilon_{m}, \alpha_{1}, \ldots, \alpha_{r}, \beta_{1}, \ldots, \beta_{s}\right)= \\
& \left(\Xi^{g_{i_{s}}, g_{j_{s}}, \beta_{s}} \circ \ldots \circ \Xi^{g_{i_{1}}, g_{j_{1}}, \beta_{1}} \circ\right. \\
& \left.\Phi^{g_{r}, \alpha_{r}} \circ \ldots \circ \Phi^{g_{1}, \alpha_{1}} \circ \Phi^{f_{m}, \varepsilon_{m}} \circ \ldots \circ \Phi^{f_{1}, \varepsilon_{1}}\right)\left(x_{*}\right) .
\end{aligned}
$$

Let $\mathcal{V}$ be the set of all indexed families $V=\left\{v_{k}\right\}_{k=1, \ldots, s}$ of vectors $v_{k} \in\left[g_{i_{k}}, g_{j_{k}}\right]\left(x_{*}\right)$. For each member $V=\left\{v_{k}\right\}_{k=1, \ldots, s}$ of $\mathcal{V}$, let $\mathcal{L}^{V}$ be the linear map from $X$ to $\mathbb{R}^{n}$ given by

$$
\begin{aligned}
& \mathcal{L}^{V}\left(\varepsilon_{1}, \ldots, \varepsilon_{m}, \alpha_{1}, \ldots, \alpha_{r}, \beta_{1}, \ldots, \beta_{s}\right) \\
= & \sum_{i=1}^{m} \varepsilon_{i} f_{i}\left(x_{*}\right)+\sum_{j=1}^{r} \alpha_{j} g_{j}\left(x_{*}\right)+\sum_{k=1}^{s} \beta_{k} v_{k} .
\end{aligned}
$$

Then $\Theta(0)=x_{*}$, and Theorems 4.1 and 5.1, together with the chain rule for GDQs (cf. [4], [5]) imply that the set $\left\{\mathcal{L}^{V}: V \in \mathcal{V}\right\}$ is a generalized differential quotient of $\Theta$ at 0 in the direction of $X$. The hypothesis of Theorem 2.1 implies that all the maps $\mathcal{L}^{V}$ are surjective. The desired conclusion then follows from the open mapping property for GDQs (Theorem 3.5).

\section{References}

[1] Kawski, M., and H.J. Sussmann, "Noncommutative power series and formal Lie-algebraic techniques in nonlinear control theory." In Operators, Systems and Linear Algebra: Three Decades of Algebraic Systems Theory, Kaiserslautern, 1997, U. Helmke, D. Praetzel-Wolters, E. Zerz Eds.; B. G. Teubner Stuttgart, 1997, pp 111-129.

[2] Pontryagin, L.S., V.G. Boltyanskii, R. V. Gamkrelidze and E.F.Mischenko, The Mathematical Theory of Optimal Processes. Wiley, New York, 1962.

[3] Sussmann, H.J., "A maximum principle for hybrid optimal control problems." In Proc. 38th IEEE Conf. Decision and Control, Phoenix, AZ, Dec. 1999. IEEE publications, New York, 1999, pp. 425-430.

[4] Sussmann, H.J., "Résultats récents sur les courbes optimales." In $15^{e}$ Journée Annuelle de la Société Mathémathique de France (SMF), Publications de la SMF, Paris, 2000, pp. 1-52.

[5] Sussmann, H.J., "New theories of set-valued differentials and new versions of the maximum principle of optimal control theory." In Nonlinear Control in the year 2000, A. Isidori, F. LamnabhiLagarrigue and W. Respondek Eds., SpringerVerlag, London, 2000, pp. 487-526.

[6] Sussmann, H.J., "Set-valued differentials and the hybrid maximum principle." In Proc. 39th IEEE Conf. Decision and Control, Sydney, Australia, December 12-15, 2000, IEEE publications, New York, 2000. 